\title{
A RAPID PROCEDURE TO MAP OVERBURDEN THICKNESSES BY USING A 3 POINT ELECTRICAL SOUNDING METHOD
}

\author{
M. Lajarthe ${ }^{1}$, N. Florsch ${ }^{1}$ et P. Andrieux ${ }^{2}$ \\ ${ }^{1}$ LEPCAT, Université de La Rochelle, Avenue Marillac, 17042 La Rochelle Cedex 01 \\ ${ }^{2}$ Département de Géophysique appliquée, UPMC, 4 Place Jussieu, 75252 Paris cedex 05 )
}

\section{INTRODUCTION}

Electrical soundings are usually used for overburden thickness estimation. Since a classical electrical sounding (herein called " complete sounding») involves at least ten or a dozen points, vast area surveying requires an important amount of time on the field.

Here, we study the possibility to reduce an electrical sounding to only three measurements (we call it the "3-point method"), but which provide the same information as would be obtained from a complete sounding.

\section{THEORY}

Assuming a two-layer model applies, it is theoretically possible to retrieve two resistivities and one thickness (i.e. 3 parameters $\rho_{\mathrm{i}}, \rho_{2}$, and h), from only three independent apparent resistivity measurements. Those usually correspond to three electrode spacings. In this study, we use the Wenner array but other arrays could alternatively be chosen. With this array, the 3 electrode spacings are $a_{1}=a_{0} / k, a_{2}=a_{0}$ and $a_{3}=k a_{0}$, where $k$ is a given factor supposed to be larger than 1 and where $a_{0}$ is taken as a reference value. Figure 1 illustrates this configuration.

\section{PARAMETERS CHOICE}

Intuitively, the central difficulty of the method lies in the choice of the parameters $a_{0}$ and $k$ relative to the field features. Indeed, to define $a_{0}$, one must take account of the depth of the interface, and $k$ should depend on the resistivity ratio $p_{2} / p_{1}$. To reach an efficient inversion, $a_{0}$ should be close to the inflexion point of the 2-layer sounding curve: a too small or too large value does not yield a good prospect of the interface depth. On the other hand, $\mathrm{k}$ must be chosen so that the two outer measurements reach the tips of the (non-measured) resistivity sounding curve: a too small or too large $\mathrm{k}$ value leads to indeterminations and, moreover, are not suitable on the field. This is illustrated qualitatively in Figure 2.

To study this problem quantitatively, we have estimated the variations of the Jacobian determinant appearing in inversions of Newton-Raphson type (Figure 3), to be a good criterion to estimate values for the parameters $\mathrm{a}_{0}$ and $\mathrm{k}$. The more this determinant approaches zero, the less stable the inversion. Hence, the parameters must be chosen in the area where the determinant is large, but the maximum value itself would be irrelevant as the thickness and the resistivities on the field are variable. Figure 3 shows plots obtained for a thickness of $1 \mathrm{~m}$, 
and $\rho 2 / \rho 1$ ratios of $10,5,1 / 10$ and $1 / 5$, which are values commonly encountered. According to these plots and for a relatively conductive superficial layer, $4<a_{0}<6$ and $k>8$ seem to be adequate values for mapping thicknesses of about $1 \mathrm{~m}$ for a resistivity contrast near 5 .

\section{FIELD EXAMPLE}

To validate the method, we have performed a set of electrical complete soundings in an area where limestone is the normal country rock with an overburden ground thickness ranging from a few decimeters to about 2 meters. Figure 5-d shows the location of these soundings on a pole-pole resistivity map realised with a $0.5 \mathrm{~m}$ electrode spacing and a $0.5 \times 0.5 \mathrm{~m}$ gridding.

Then we have interpreted those soundings according to a two-layer model, first with the classical method, that is, by means of " master curves» with the whole set of apparent resistivity measurements for each complete sounding, and second, independently, by using the "3-point method ", that is, for each of these soundings, by inverting only the three resistivity values, measured for the electrode spacing $a_{1}=a_{0} / k, a_{2}=a_{0}$ and $a_{3}=k a_{0}$.

The choice of $a_{0}$ and $k$ requires a previous estimation of both resistivity ratio and thickness. As the latter is variable in space (from point to point), it is judicious to choose a representative value of the mean estimated thickness and of the Wenner array investigation depth. In nearby trenches dug for archaeological investigation, this mean thickness was about $1 \mathrm{~m}$, and the resistivity contrast did not exceed a factor of 10 . The best parameter values would thus seem to be $a_{0}=5$ and $k=8$ (cf. figure 3 ), but in practice, the estimation of the mean thickness and resistivity ratio may be of much lower quality. Hence, to simulate a more realistic case, we purposely chose values of $\mathrm{a}_{0}=2$ and $\mathrm{k}=5$, outside the optimum area of the determinant.

Figure 5 summarises the results obtained both with the " 3 -point inversion method " (schemes bl to b10) and with classical one (schemes a1 to a10), shown together for comparison. Differences in results, are virtually negligible, thereby showing that the " 3 -point method » is a satisfactory alternative to complete sounding.

\section{EXTENSION : BAYESIAN APPROACH}

We also applied a Bayesian approach to invert the «3-point» data. Figure 4-b shows the results obtained for sounding $n^{\circ} 8$. Despite the unfavourable values chosen for $a_{0}$ and $k$, the 2 layer parameters are well determined. However, for a few other soundings, especially when the overburden thickness is far from the mean value a priori expected, one or two of the parameters are less well constrained, as illustrated on figure 4-a for sounding $n^{\circ} 1$.

\section{CONCLUSION}

The « 3-point method» presented here is a suitable procedure to map the overburden thickness and resistivities for a 2-layer model, once relevant values for the geometric parameters $a_{0}$ and $k$ have been obtained from a few complete soundings, or else .

\section{ACKNOWLEDGMENTS}

We wish to thank J-C. C. Mercier for his contribution to the last version of this note. 


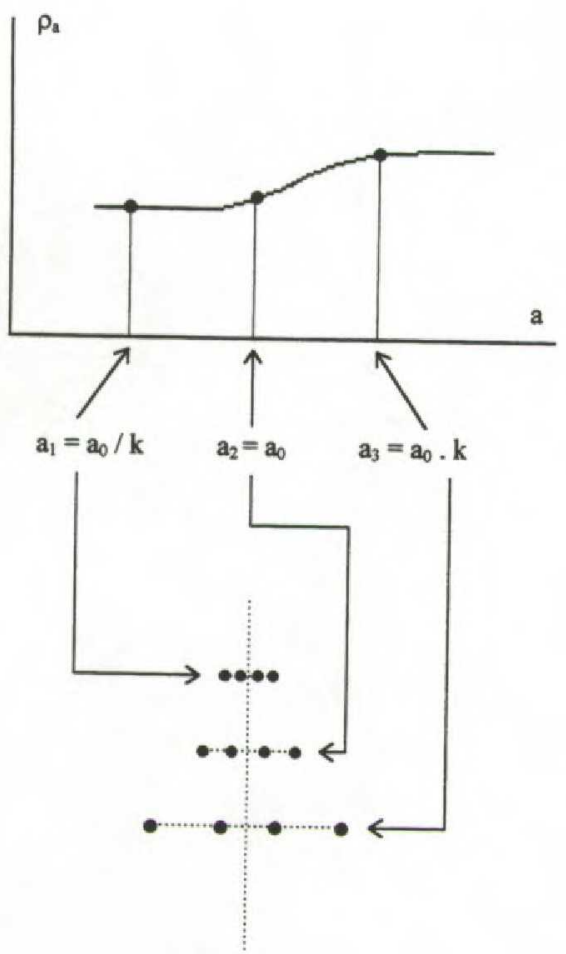

figure 1

The « 3-point method » system (example with $\mathrm{a}_{0}=1$ and $\mathrm{k}=2$ )

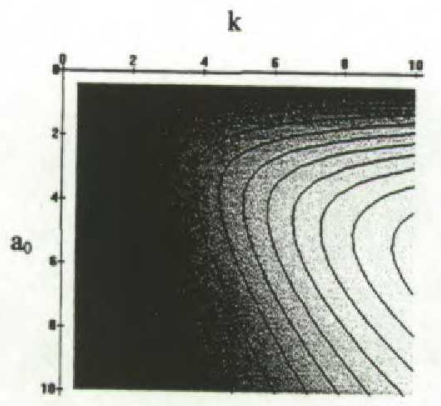

$\rho_{1}=10-\rho_{2}=100-\mathrm{h}=1$

k

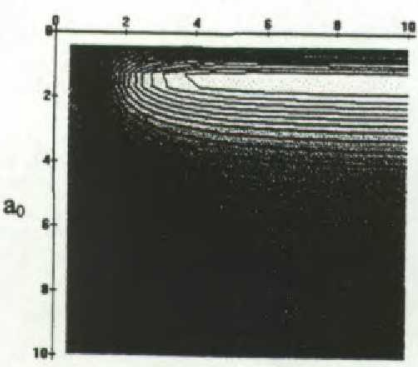

$\rho_{1}=100-\rho_{2}=10-h=1$

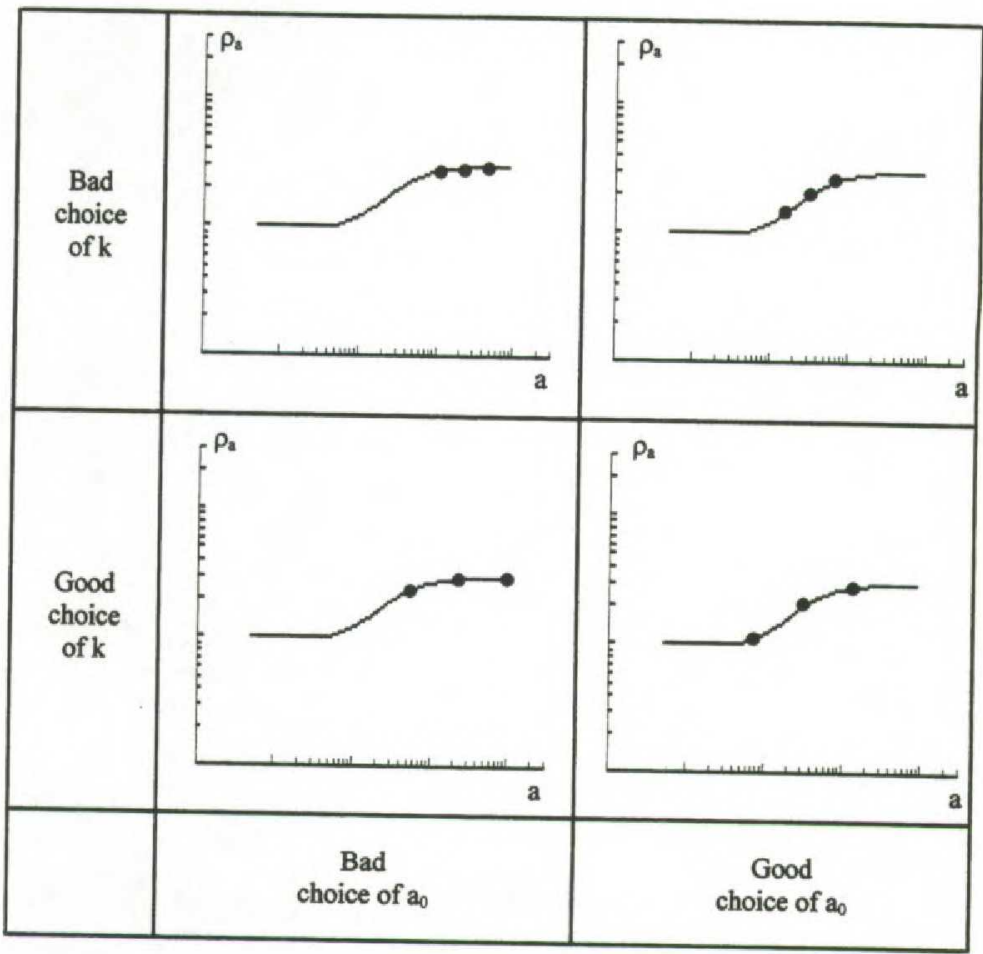

figure 2

How to choose parameters $a_{0}$ et $k$ $a_{0}$
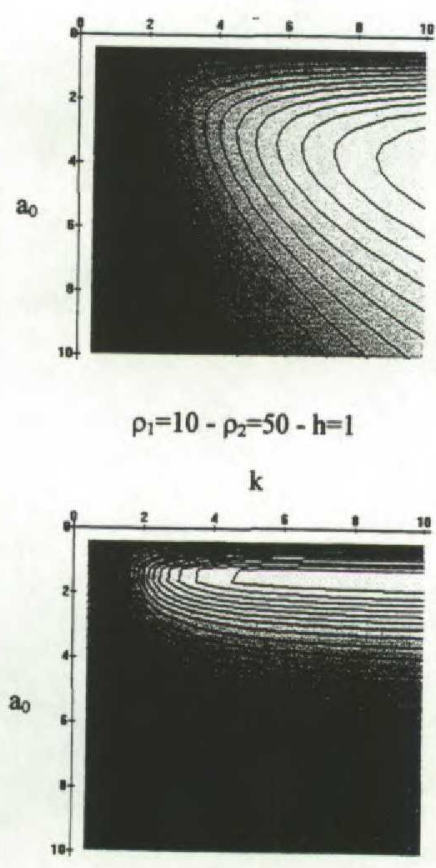

$\rho_{1}=50-\rho_{2}=10-h=1$

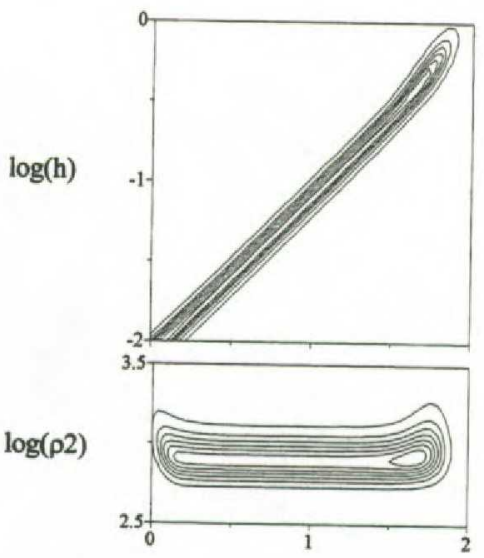

(a)

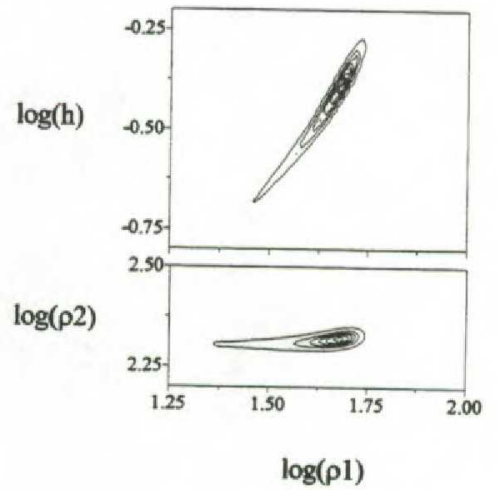

(b)

figure 4

Results of Bayesian inversion for soundings 1 (a) and 8 (b) 


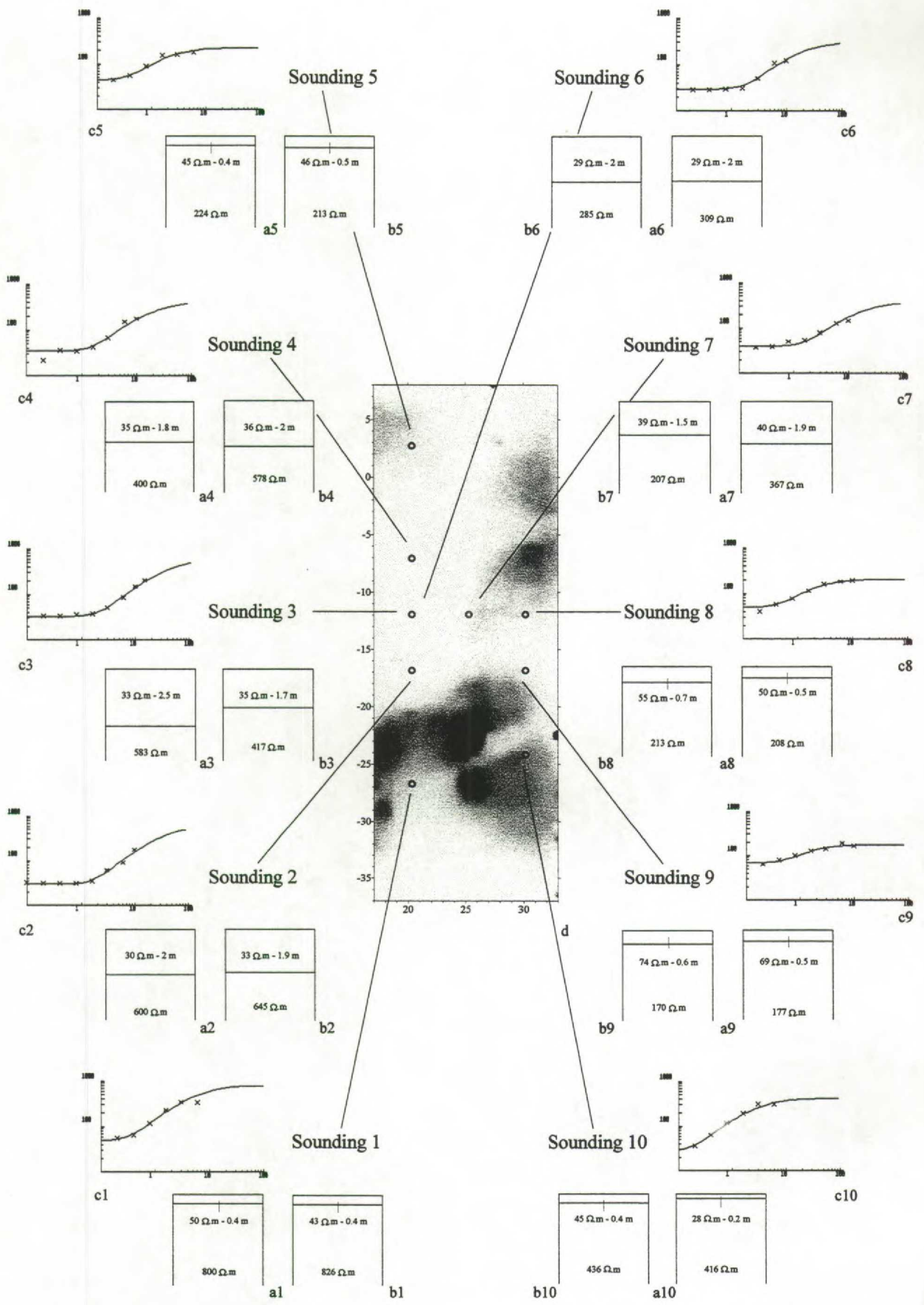

figure 5: Sounding positions (d), Sounding curves (c1-c10), interpretations of classical method results (a1-a10), and « 3-point method » results (b1-b10) as 2-layers models. 